\title{
P-Loop Channels: Experimental Structures, and Physics-Based and Neural Networks-Based Models
}

\author{
Denis B. Tikhonov * ${ }^{\mathbb{D}}$ and Boris S. Zhorov
}

Citation: Tikhonov, D.B.; Zhorov, B.S. P-Loop Channels: Experimental Structures, and Physics-Based and Neural Networks-Based Models. Membranes 2022, 12, 229. https:/ / doi.org/10.3390/membranes12020229

Academic Editor: Tanima Bose

Received: 27 December 2021

Accepted: 9 February 2022

Published: 16 February 2022

Publisher's Note: MDPI stays neutral with regard to jurisdictional claims in published maps and institutional affiliations.

Copyright: () 2022 by the authors Licensee MDPI, Basel, Switzerland. This article is an open access article distributed under the terms and conditions of the Creative Commons Attribution (CC BY) license (https:// creativecommons.org/licenses/by/ $4.0 /)$.

\author{
I.M. Sechenov Institute of Evolutionary Physiology and Biochemistry, Russian Academy of Sciences, \\ 194223 St. Petersburg, Russia; boriszhorov@yahoo.com \\ * Correspondence: denistikhonov2002@yahoo.com
}

\begin{abstract}
The superfamily of P-loop channels includes potassium, sodium, and calcium channels, as well as TRP channels and ionotropic glutamate receptors. A rapidly increasing number of crystal and cryo-EM structures have revealed conserved and variable elements of the channel structures. Intriguing differences are seen in transmembrane helices of channels, which may include $\pi$-helical bulges. The bulges reorient residues in the helices and thus strongly affect their intersegment contacts and patterns of ligand-sensing residues. Comparison of the experimental structures suggests that some $\pi$-bulges are dynamic: they may appear and disappear upon channel gating and ligand binding. The AlphaFold 2 models represent a recent breakthrough in the computational prediction of protein structures. We compared some crystal and cryo-EM structures of P-loop channels with respective AlphaFold2 models. Folding of the regions, which are resolved experimentally, is generally similar to that predicted in the AlphaFold2 models. The models also reproduce some subtle but significant differences between various P-loop channels. However, patterns of $\pi$-bulges do not necessarily coincide in the experimental and AlphaFold2 structures. Given the importance of dynamic $\pi$-bulges, further studies involving experimental and theoretical approaches are necessary to understand the cause of the discrepancy.
\end{abstract}

Keywords: ligand-channel interactions; sequence alignment; $\pi$-bulges; crystal structures; cryo-EM structures; potassium channels; sodium channels; calcium channels; TRP channels; ionotropic glutamate receptors

\section{Introduction}

Among the various families of ion channels, tetrameric P-loop channels stand alone due to their large functional diversity and importance in physiology, pathophysiology, pharmacology, and toxicology [1-6]. This superfamily includes various voltage- and ligandgated potassium channels, voltage-gated sodium and calcium channels, TRP (transient receptor potential) channels, and ionotropic glutamate receptors [7-10]. The common structural motif of all the family members is the pore-forming domain (PD). The latter contains the outer and inner transmembrane helices linked by a membrane re-entrant P-loop with a membrane-descending pore helix (P-helix). The C-ends of the four P-helices with few residues at the $\mathrm{C}$-end converge to the pore axis to form a pore that narrows with the selectivity filter (SF) (Figure 1). The activation mechanisms of P-loop channels are very different due to specific structural and functional properties of the gating-control domains, which are attached to the pore domain [11-16].

The P-loop channels include tetramers (glutamate receptors, TRP, and potassium channels), dimers of dimers (two-pore channels) [17], and pseudo-tetramers. The latter are eukaryotic sodium and calcium channels in which large pore-forming subunit folds from a single polypeptide chain of four homologous repeats [1,2]. Interestingly, even the orientation of P-loops in the membrane is not conserved in P-loop channels-in ionotropic glutamate receptors, the P-loops are partially exposed to cytoplasm, whereas in other members of the superfamily, the P-loops are partially exposed to the extracellular space $[18,19]$. 


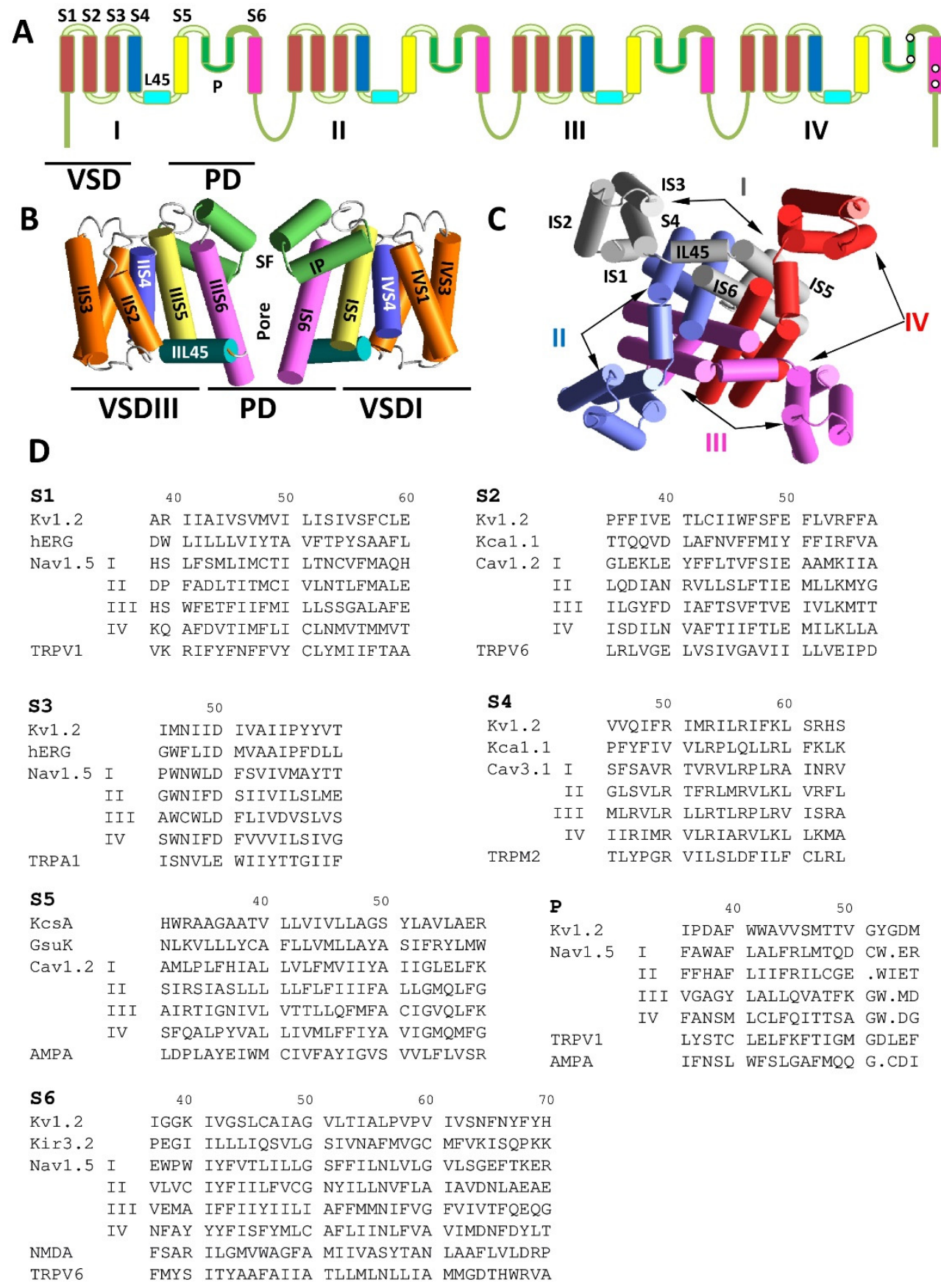

Figure 1. Transmembrane topology and folding of some P-loop channels. (A) Transmembrane topology of a eukaryotic sodium and calcium channel. Roman numerals indicate repeats in heterotetrameric channels. Voltage-sensing domains (VSD) and pore domain (PD) are marked. (B) Side view of the NavAb X-ray structure (3rvy). Only two repeats are shown for clarity. The segments are colored as in A. The P-loops have the selectivity filter (SF). (C) Intracellular view of the NavAb crystal structure with P-loops removed for clarity. Individual subunit repeats are shown with different colors. All four repeats contribute to the PD that surrounds the central pore, whereas the VSDs are localized peripherally. (D) Aligned sequences of helical segments some P-loop channels. The alignment is based on three-dimensional (3D) positions and orientations of residues in experimental structures.

Figure $1 \mathrm{~A}-\mathrm{C}$ shows the organization of eukaryotic voltage-gated sodium and calcium channels according to the structural data. The alpha subunit is assembled from homologous repeats I-IV. Each repeat has the VSD, which includes transmembrane segments S1-S4, and 
contributes a quarter to the PD (segments S5, P, and S6). The PD surrounds the central pore, whereas VSDs are localized peripherally (Figure 1B,C).

The similar architecture of the PD in P-loop channels was recognized before the crystal structures had become available. Location of the activation gate at the cytoplasmic part of the pore [20], location of the selectivity filter at the P-loop turn [21], and the clockwise arrangement of repeats I, II, III, and IV in the sodium channels [22] were determined by experimental studies, which included mutagenesis, electrophysiology, and analysis of drug and toxin action.

P-loop channels are intensively studied due to their key roles in multiple physiological and pathophysiological processes and their importance in pharmacology and toxicology. Various characteristics of P-loop channels, including 3D structures, ion permeation and gating, impact of disease mutations, and modulation by drugs and toxins are described in many recent reviews, e.g., [2,3,7-10]. The vast majority of the reviews are focused on specific channel subfamilies, and they only rarely provide comparison with channels from other subfamilies. This is not surprising given the huge functional diversity of the P-loop channels and the distinct roles of individual subfamilies in physiology, pathophysiology, pharmacology, and toxicology. The similar architecture of the pore-forming domain of different P-loop channels calls for a comparative analysis of typical representatives from different channel subfamilies. Such comparison, which may provide novel structural and functional insights, is a major goal of the current review.

We describe crystal and cryo-EM (cryo-electron microscopy) structures and physicsbased models of various P-loop channels. We further compare the experimental structures of several channels and their recent AlphaFold2 models. The comparison reveals generally similar folding, but also differences in some important details. In particular, the inner helices of many P-loop channels have $\pi$-bulges, which appear and disappear depending on the channel's functional state and even on the presence of ligands. The patterns of $\pi$-bulges do not always coincide in experimental structures and AlphaFold2 models.

\section{Comparative Structural Analysis of P-Loop Channels}

To facilitate comparison of various P-loop channels, we use here a modified labeling scheme, which is universal for P-loop channels [18]. The most sequentially conserved and functionally important residues in transmembrane helices S1-S6 and in P-loops are assigned the number 50 (Figure 1D). A residue label includes the segment designation and a relative residue number in the segment. The $\mathrm{C}$-terminal part of the outer helices (S5) has a small residue (G, A, or S), which is involved in a knob-into-hole contact with a bulky residue at the N-end of P-helix of the same subunit/repeat [23]. The small residue is labeled S5/50. For example, in the Cav1.2 channel, alanine in the first repeat of the outer helix (IS5) is labeled $\mathrm{A}^{\mathrm{IS5} / 50}$ (Figure 1D). Label $\mathrm{P} / 50$ is assigned to the selectivity-filter residues in P-loops, which include valine in the TVGYG fingerprint sequence of potassium channels [24], DEKA ring in eukaryotic sodium channels [21], and EEEE ring in eukaryotic calcium channels [25]. The gating hinge glycine in the inner helix of potassium channels [26] and sequentially matching residues in other channels are labeled S6/50. It should be noted that the sequence alignment shown in Figure 2D does not necessarily match alignments proposed by web servers, e.g., Clustal Omega [27].

In order to obtain three-dimensional (3D) alignment P-loop channels, we used as a template the chimeric potassium channel Kv1.2/Kv2.1 (2r9r), the first eukaryotic P-loop channel whose crystal structure was obtained with the resolution below $2.5 \AA$ [28]. The channel was oriented so that the pore axis coincided with the $z$-axis, the tyrosine backbone CA atoms in selectivity filter GYG motif laid in plane $x O z$, and axis $O x$ was directed towards the CA atom of the GYG tyrosine in subunit III. Other P-loop channels were 3D-aligned with the Kv1.2/Kv2.1 channel by minimizing RMS deviations of the CA atoms in positions p38-p47 of four $\mathrm{P}$ helices, which are the most 3D-conserved segments of P-loop channels [29]. 
The list of structures used in the study is given in Table 1. The original structures are downloaded from the protein data bank (PDB) (www.rcsb.org, accessed on 25 December 2021) and the AlphaFold server (www.alphafold.ebi.ac.uk, accessed on 25 December 2021).

A
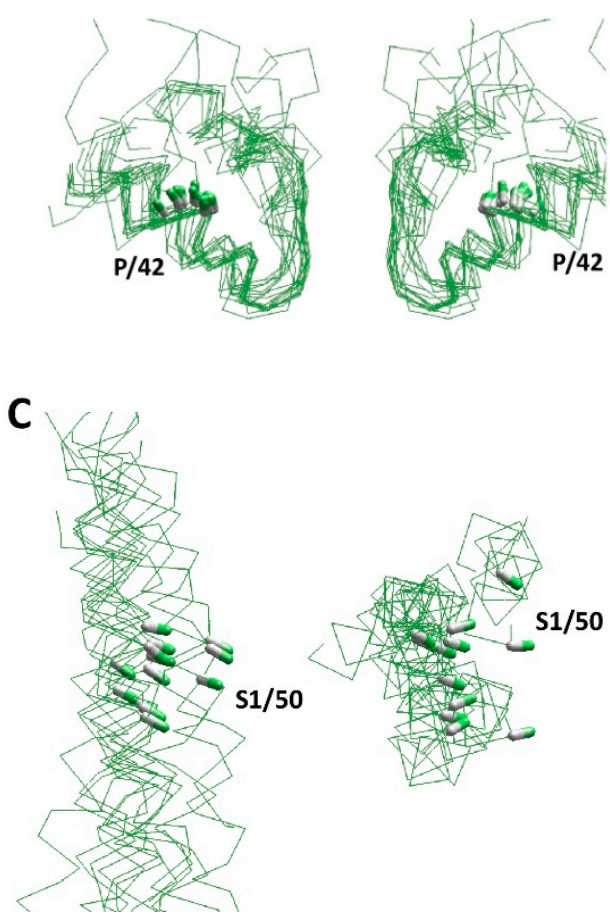

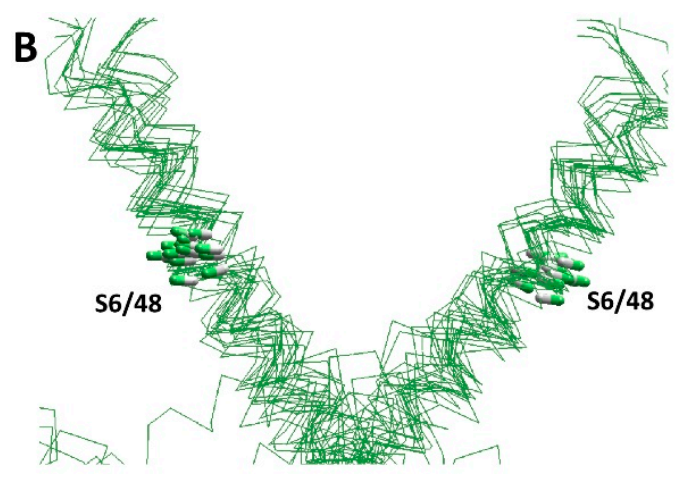

Figure 2. Conserved folding of experimental structures of P-loop channels. Individual segments are shown for the 3D-aligned structures of the following channels: KcsA (1bl8), Kv1.2-Kv2.1 (2r9r), MlotiK1 (3beh), TRPV1 (3j5r), TRPA1 (3j9p), NavAb (3rvy), GsuK (4gx5), Kir3.2 (4kfm), Slo (5tj6), hERG (5va2), NavPaS (5x0m), rbCav1.1 (6byo), TPC1 (6c96), NMDA (6cna), AMPA (6dm0), Cav3.1 (6kzp), TRPM2 (6mj2), Nav1.5 (6uz3), and TRPV6 (6e2g). CA-CB bonds of residues in matching positions of the sequence alignments (Figure 1D) are shown by sticks. (A) Side view of P-loops. (B) Side view of $\mathrm{S} 6$ helices. Despite significant sequential, functional, and structural diversity of the channels, the positions and orientations of the $\mathrm{CA}-\mathrm{CB}$ bonds are very similar in P-loops and $\mathrm{S} 6$ helices. (C) Side (left panel) and extracellular (right panel) views of S1 helices. Despite the similarity of VSD backbones in the structures, which were 3D-aligned by minimizing RMS deviations of P-helices, is less obvious, the positions and orientations of the $\mathrm{CA}-\mathrm{CB}$ bonds of residues $\mathrm{S} 1 / 50$ are comparable.

Table 1. Ion channel structures discussed in this work.

\begin{tabular}{|c|c|c|c|c|c|c|c|}
\hline Channel & PDB ID & Ref. & $\mathbf{A F}^{\mathrm{a}}$ & Channel & PDB ID & Ref. & $\mathbf{A F}^{\mathrm{a}}$ \\
\hline Potassium & & & & Calcium & & & \\
\hline $\mathrm{K} \operatorname{csA}$ & $1 \mathrm{bl} 8$ & [26] & & rbCav1.1 & $5 \mathrm{gjv}$ & [30] & \\
\hline MthK & $6 u 6 e$ & [31] & & & 6jpa & [32] & \\
\hline MlotiK1 & 3beh & [33] & & & 6jpb & [32] & \\
\hline Kv1.2/Kv2.1 & $2 \mathrm{r} 9 \mathrm{r}$ & [28] & & & 7jpk & [34] & \\
\hline hKv1.2 & & & P16389 & & 7jpw & [34] & \\
\hline hKv1.6 & & & P17658 & & 6jp5 & [32] & \\
\hline hKv2.1 & & & Q14721 & hCav1.1 & & & Q13698 \\
\hline hKv3.1 & & & P48547 & hCav1.3 & & & Q01668 \\
\hline hKv7.1 & 6uzz & [35] & P51787 & hCav3.1 & $6 \mathrm{kzo}$ & [36] & O43497 \\
\hline hERG & $5 \mathrm{va} 2$ & [37] & & & $6 \mathrm{kzp}$ & [36] & \\
\hline Kir3.2 & $4 \mathrm{kfm}$ & [38] & & hCav3.2 & & & O95180 \\
\hline
\end{tabular}


Table 1. Cont.

\begin{tabular}{|c|c|c|c|c|c|c|c|}
\hline Channel & PDB ID & Ref. & $\mathrm{AF}^{\mathrm{a}}$ & Channel & PDB ID & Ref. & $\mathrm{AF}^{\mathrm{a}}$ \\
\hline GsuK & $4 g \times 5$ & [39] & & hCav1.4 & & & O60840 \\
\hline Slo & 5 tj6 & [40] & & & & & \\
\hline Sodium & & & & iGluR & & & \\
\hline \multirow[t]{5}{*}{ NavAb } & 3rvy & [41] & & AMPA & $6 \mathrm{dm} 0$ & [42] & \\
\hline & $5 \mathrm{vb} 2$ & [43] & & NMDA & $6 c n a$ & [44] & \\
\hline & $5 \mathrm{vb8}$ & [43] & & & & & \\
\hline & $6 p 6 x$ & [45] & & TRP & & & \\
\hline & $6 p w p$ & [45] & & TRPA1 & $3 j 9 p$ & [46] & O75762 \\
\hline NavMs & $4 \mathrm{f} 41$ & [47] & & TRPV1 & $3 j 5 r$ & [48] & Q8NER1 \\
\hline NavRh & $4 \mathrm{~d} \times w$ & [49] & & TRPV2 & 6007 & [50] & Q9Y5S1 \\
\hline NavPaS & $6 a 95$ & [51] & & TRPV3 & $6 \mathrm{dvy}$ & [52] & Q8NET8 \\
\hline Nav1.2 & $6 \mathrm{j} 8 \mathrm{e}$ & [53] & Q01118 & & $6 \mathrm{mhw}$ & [54] & \\
\hline hNav1.4 & 6agf & [55] & P35499 & & $6 \mathrm{mho}$ & [54] & \\
\hline EeNav1.4 & $5 x s y$ & [56] & & & $6 p v l$ & [57] & \\
\hline \multirow[t]{3}{*}{ rNav1.5 } & 6uz3 & [58] & P15389 & & $6 \mathrm{dvw}$ & [52] & \\
\hline & $7 \mathrm{fbs}$ & [59] & & & 6uw9 & [60] & \\
\hline & $7 \mathrm{k} 18$ & [61] & & & $6 \lg p$ & [62] & \\
\hline hNav1.5 & 6 lqa & [63] & Q14524 & & 6uw4 & [60] & \\
\hline \multirow[t]{2}{*}{ hNav1.7 } & $6 \mathrm{j} 8 \mathrm{j}$ & [64] & & & $6 \mathrm{mhs}$ & [54] & \\
\hline & $6 n 4 r$ & [65] & & TRPV6 & 5iwk & [66] & \\
\hline hNav1.9 & & & Q9UI33 & & $6 \mathrm{e} 2 \mathrm{~g}$ & [67] & Q9H1D0 \\
\hline hNav2.1 & & & Q01118 & TRPM2 & $6 \mathrm{mj} 2$ & [68] & O94759 \\
\hline TPC1 & $6 c 96$ & [69] & & TRPM6 & 6 Bo 9 & [70] & \\
\hline
\end{tabular}

a UniProt accession code of neural network-predicted structures of proteins deposited in the AlphaFold protein structure database https: / / alphafold.ebi.ac.uk, accessed on 25 December 2021 [71,72].

\section{Crystal and Cryo-EM Structures}

The KcsA potassium channel from the soil bacterium Streptomyces lividans was the first P-loop channel whose 3D structure was obtained with X-ray crystallography [26]. The crystal structures of homotetrameric potassium channels served as templates for homology modeling of eukaryotic sodium, calcium, and glutamate receptor channels [73-80]. The similarity between potassium channels and other P-loop channels was often implied to rationalize experimental data (e.g., mutational analysis and drug action), even without building homology models [81-83].

In agreement with an earlier concept of the open-gate architecture, which was based on indirect experimental data, the crystal structure of the calcium-gated potassium channel MthK revealed diverging C-parts of the inner helices exposed to the intracellular vestibule [84]. Although the open Kv1.2 channel has different conformation of the S6 helices [85], the principal gating mechanism is the same. In both channels, the opening involves shifting and twisting C-terminal parts of the S6 helices due to conformational changes at the conserved gating-hinge glycine residues. Comparison of the open-gate structure of the mammalian Kv1.2 channel and closed-gate structure of a bacterial cyclic nucleotide-regulated channel MlotiK1 [33] revealed the critical role of the S4-S5 linker helices that form a cuff around the bundle of S6 helices and limit their movement during gating.

The activation gating mechanism involving diverging inner helices is common for Ploop channels. Specific examples are available for almost every member of the superfamily. It was further demonstrated that the selectivity-filter region of the KcsA channel operates as the C-type slow-inactivation gate $[86,87]$. Rearrangements at the selectivity-filter region that can prevent the ion flow were also reported for other P-loop channels, including Kv7.1 [88], TRPV1 [48], Nav1.4 [89], Cav1.2 [90], and glutamate receptor [91] channels.

Over the past two decades, numerous experimental structures of various P-loop channels have been published (Table 1 and references therein). The structures have confirmed the conserved general architecture of various channels. Figure 2 shows several segments 
in the 3D-aligned experimental structures of nineteen P-loop channels, which represent potassium, sodium, and calcium channels, as well as TRP channels, two-pore channels, and ionotropic glutamate receptors. The channels have different functional properties, ion selectivity, gating mechanisms, and length and folding of intra- and extracellular loops. Despite these differences, the positions and orientation of the CA-CB bonds in sequentially matching positions of P-loops and S6 helices are very similar (Figure 2B). While the structural similarity of $\mathrm{S} 1$ helices in the voltage-sensing domain is less obvious, the positions and orientations of the CA-CB bonds in residues S1/50 are rather similar (Figure 2C). This overall similarity validates the comparative structural analysis of different P-loop channels in different functional states, despite the sequence alignment not always being obvious (Figure 1D).

The experimental 3D structures have shed light on the common and specific features of different P-loop channels and greatly advanced our understanding of the mechanisms of ion permeation, selectivity, gating, and sensitivity to drugs and toxins $[3,9,92,93]$. The vast majority of experimental structures show P-loop channels in the energetically mostpreferred states, with the pore domain in a presumably inactivated state and VSDs in the activated states, e.g., [58]. Notable exceptions include the cryo-EM structures of eukaryotic sodium channels with the open pore domain [59] or with VSDs deactivated by proteinaceous toxins $[61,65]$. The crystal structures of the NavAb channel, in which engineered disulfide bridges stabilize the pore domain in the closed or open states [43] or VSDs in the deactivated state [45], provide important templates for modeling eukaryotic channels in sparsely populated, but functionally important conformations. These structures were used, in particular, to build models of the medically important channels hCav1.2 and hNav1.5 in different states, to map state-dependent contacts, and to suggest the structural mechanisms of dysfunction of the channels' disease variants [94-96].

The available 3D structures have opened an avenue for computational studies that have addressed important problems, which currently cannot be resolved experimentally. Examples include MD simulations of ion permeation through prokaryotic potassium channels [97-99], MD simulation of voltage-sensing gating with the supercomputer Anton [100], and computational studies of ion selectivity in potassium [101-103] and sodium [104-107] channels.

Besides the conserved structural and functional features of P-loop channels, experimental structures have revealed important differences between different channel types. The crystal structures of the prokaryotic sodium channels NavAb with the closed pore [41], NavMs with the open pore [47], and NavRh in a presumably inactivated state [49] revealed their differences with potassium channels not only in the selectivity-filter region, but also in the pore domain architecture. Particularly, while the P-loop of potassium channels has only one helix (a membrane-descending P-helix), P-loops on sodium and calcium channels have two helices separated by the selectivity-filter region: a membrane-descending P1-helix at the $\mathrm{N}$-terminal part of the P-loop and a membrane-ascending helix P2. Furthermore, subunit interfaces in sodium channels are significantly wider than those in potassium channels.

Experimental 3D structures have led to the significant modification of some structural concepts on P-loop channels. Thus, the ball-and-chain mechanism of the fast (N-type) inactivation of voltage-gated potassium and sodium channels was proposed to involve a short fast-inactivated particle at the channel cytoplasmic part to enter the open pore and physically occlude the ion permeation pathway [108-110]. However, the cryo-EM structures of the electric eel channel Nav1.4 [56] and other eukaryotic sodium channels show that the fast-inactivation LFM (IFM) motif in the intracellular linker between repeats III and IV does not block the pore, but binds between helices IIIS4-S5 and IIIS5 and the membrane-oriented faces of helices IIIS6 and IVS6. Another example is the 3D structures of glutamate receptor channels, wherein early X-ray structures suggested that despite the general overall similarity, glutamate-gated channels differ significantly from potassium channels. However, recent studies, which employed improved experimental methods, demonstrated a high similarity between these subfamilies of P-loop channels [111]. 


\section{Structures of P-Loop Channels with Drugs and Toxins}

P-loop channels are targets for numerous naturally occurring toxins [112], medically important and illicit drugs [113,114], and insecticides [115]. Theoretical and experimental studies greatly advanced our understanding of the ion channels' sensitivity to various ligands. Before the experimental structures of ligand-channel complexes became available, homology models based on the crystal structures of potassium channels and ligand docking methods were used to rationalize, in structural terms, large experimental data accumulated over decades of intensive research. These computational studies employed data from mutational, electrophysiological, and ligand-binding experiments, which determined ligand-binding binding regions and key ligand-channel contacts, e.g., [75,116]. The next generation of more precise ligand-channel models is based on crystal and cryo-EM structures of corresponding channels or their close homologs. Such models have been used not only to rationalize the available experimental data, but also to predict the structures of new ligands, e.g., [117,118].

The experimental structures of ligand-channel complexes have allowed for estimating the predictive power of early models. For example, homology models of the NMDA (N-methyl-D-aspartate) and AMPA ( $\alpha$-amino-3-hydroxy-5-methyl-4-isoxazolepropionic acid) glutamate receptor channels with ligands were published long before the first experimental structures of the respective complexes had become available. The experimental structures confirmed major aspects of the model-based predictions, as seen in [111] and the references therein.

Drug binding to different regions of sodium channels was addressed in many studies. Initially, homology models based on the crystal structures of potassium channels were used to dock important drugs, including local anesthetics and other inner pore blockers $[75,76]$. Small molecular ligands were proposed to reach the binding site in the inner pore through the interface between neighboring S6 helices [119,120]. These models provided the first structural visualization for the hypothesis that local anesthetics and other small molecule drugs can reach the inner pore of the closed sodium channel through a hydrophobic access pathway [121]. Later, the crystal structure of the NavAb channel demonstrated that fenestrations between the $\mathrm{S} 6$ helices in the sodium channel are much wider than those in potassium channels [41]. Experimental structures of sodium channels were used to elaborate a new generation of models to explain the action of local anesthetics and related drugs [122-125]. Recent crystal and cryo-EM structures of sodium channels with drugs in the inner pore $[58,63]$ are consistent with the earlier proposed models of ligandchannel interactions.

Naturally occurring toxins, including tetrodotoxin, saxitoxin, and mu-conotoxins, which bind to the outer pores of sodium channels, have been used to map their binding sites and understand the basic features of the toxin-channel interactions. Early models, which employed structure-activity data on the toxins and mutational analysis of the channels, predicted the binding sites of the toxins and their orientation in the Nav1.4 channel $[76,126,127]$. Following publication of the NavAb channel crystal structure [41], more accurate models of toxin-bound channels have been elaborated $[29,116,128-130]$. Recent cryo-EM structures of toxin-bound eukaryotic sodium channels demonstrated both achievements and limitations of the homology models [51,53,64].

Drugs that target L-type calcium channels, including phenylalkylamines, benzothiazepines, and dihydropyridines, are used to treat cardiovascular diseases [131]. Previous studies identified amino acid residues whose mutations affect the action of these drugs $[82,132]$. These data were used to build drug-bound models of the Cav1.2 channel $[74,80,133-136]$. The cryo-EM structure of the calcium channel Cav1.1 highlighted the DHP (dihydropyridines) binding region [34], which was previously revealed in intensive mutational studies and visualized in homology models.

Despite the impressive progress in the structural biology of P-loop channels, some problems remain unresolved. Importantly, the static crystal and cryo-EM structures correspond to the lowest-energy state. Thus, mechanisms of state-dependent drug binding and 
the impact of ligands on the channel transitions between functional states remain matters of speculation. For example, the cryo-EM structures of the L-type calcium channel with DHP agonists and antagonists are virtually the same and do not explain the principally different action of these ligands [34]. Another limitation of experimental structures is that ions, water molecules, lipids, and detergent molecules, which are not always resolved, may affect ligand binding poses. Therefore, the physiological relevance of the experimental structures is not unquestionable. An example is the cryo-EM structure of the L-type calcium channel with verapamil [32], where lipids and detergent molecules strongly interact with the drug and can affect the drug-channel structure.

\section{5. $\pi$-Bulges in the Inner Helices}

Atomic-scale structures have revealed some interesting features of channels that were not considered before. Thus, the inner helices in some channels are not entirely alpha helical, but they contain $\pi$-helical elements. An extra residue per helical turn in a $\pi$-bulge causes the reorientation of upstream or downstream residues by about 90 degrees as compared to the classical alpha helix. This, in turn, dramatically changes the pattern of inter-segment contacts involving the reoriented part of the helix and alters the pattern of pore-facing residues and ligand-channel contacts. For example, insertion of exceptionally conserved asparagine residues in the inner helices of sodium and calcium channels, which form state-dependent inter-segment $\mathrm{H}$-bonds and stabilize the open conformation of the pore domain $[137,138]$, may explain the evolutionary appearance of $\pi$-bulges in these channels, as well as in TRP channels. Accommodation of the additional residue would reorient the C-terminal parts of the inner helixes, which contain the activation gate residues and other residues involved in important inter-segment contact. The $\pi$-bulges preserve the orientation of residues beyond the $\pi$-helical segment and their intersegment contacts and thus provide structural tolerance to the insertions [139].

Different structures of the same P-loop channel may have different $\pi$-bulges, suggesting their dynamic nature. Some structures demonstrate structural rearrangements due to $\pi$-bulges, which are apparently induced by ligand binding. Examples include the hCav3.1 channel in the apo-state $(6 \mathrm{kzo})$ and in the complex with specific T-type channel blocker Z944 (6kzp), as well as rbCav1.1 channel structures with verapamil (6jpa) or diltiazem (6jpb). Besides affecting ligand-channel interactions, $\pi$-bulges may substantially change contacts between the S6, S5, and S4-S5 helices [139], and thus change the structural stability of the pore domain and transitions between the channel functional states.

Inner helices in TRP channels show a large diversity of $\pi$-bulges $[140,141]$. Inner helices in the same-type TRPV3 channels either lack $\pi$-bulges (6dvy, 6mhw, 6mho, 6pvl, 6dvw, and 6uw9) or have them (6lgp, 6uw4, 6vpo, and 6mhs). Such variations imply that dynamic $\pi$ bulges may govern conformational rearrangements of the inner helices. Indeed, comparison of the TRPM6 channel structures indicates that the S6 segments undergo a conformational transition from the $\alpha$-helix to $\pi$-helix upon the channel opening [70]. In TRPV1 and TRPV2 channels, $\alpha$-helical S6s and S6 helices with energetically less favorable $\pi$-bulges may represent different functional states of the channels [50]. Class II and Class III structures of the channel rbCav1.1 indicate that transition of the IIIS6 helix from conformation with the $\pi$-helical elements to the alpha-helical conformation is associated the outward motion and axial rotation of the helix [32]. Vanishing $\pi$-bulges are associated with the activation gate widening in Cav1.1, but the gate narrowing in TRPV6. Thus, clear relations between the activated gate dimensions and presence of $\pi$-bulges are lacking. The $\pi$-bulges may govern the bending of S6 helices in the channels without the glycine gating hinges, which are present in potassium channels.

State-dependent and drug-induced $\pi$-bulges, which are seen in some cryo-EM structures, suggest an unusual mechanism by which ligands may affect the channel gating. For example, the sodium channel activators batrachotoxin and veratridine and the DHP agonists and antagonists of the L-type calcium channels change probabilities of the open and closed channel states [82,142-145]. Cryo-EM structures of the Cav1.1 channel show DHP 
agonists (7jpk) and antagonists (7jpw) bound in the III/IV fenestration [34], but changes in the activation gate region are very small and do not explain the principle mechanism of action of these important ligands. Drug-induced $\pi$-bulges could reorient the $S 6$ residues and thus affect stabilities of the open- and closed-gate conformations.

\section{AlphaFold2 Models and Experimental Structures}

A recent breakthrough in structural biology is based on computational approaches, which combine artificial intelligence and energy optimization. The AlphaFold2 neural network predicted the 3D structures of all proteins in humans and 20 model organisms [71,146]. The RoseTTAFold server [147] is another major resource based on artificial intelligence. Although models of transmembrane proteins are cautioned to be of limited precision, the superposition of the hNav1.5 cryo-EM structure (6lqa) with the AlphaFold2 structure (Figure 3) shows an impressive similarity of the transmembrane and extracellular segments. The AlphaFold2 structure also shows some structured segments in the cytoplasmic parts that are not resolved in the cryo-EM structure. Below we compare some crystal and cryo-EM structures with the respective AlphaFold2 models.

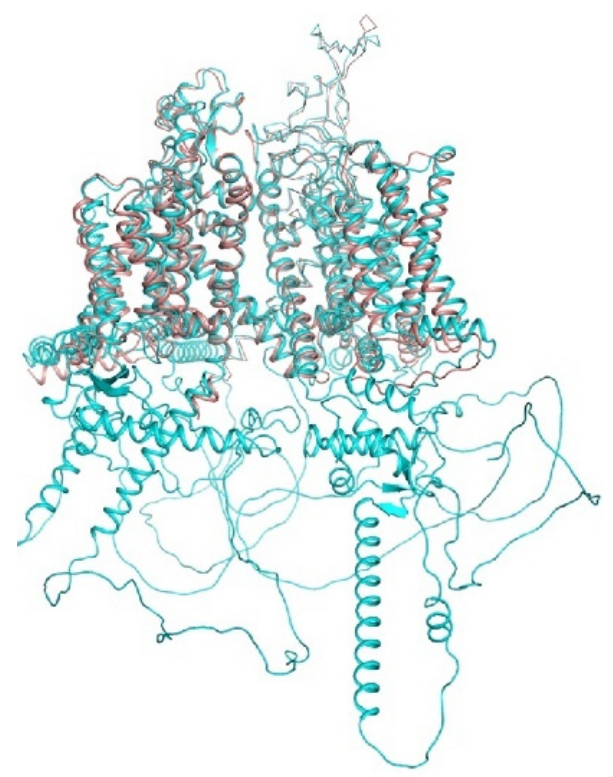

Figure 3. hNav1.5 channel. In the AlphaFold2 structure (Q14524, cyan) and cryo-EM structure (6LQA; brown), transmembrane and extracellular segments are similar. However, the AIphaFold2 structure also shows some structured segments in the cytoplasmic parts, which are not resolved in the cryo-EM structure.

Superposition of five experimental structures of voltage-gated sodium channels (hNav1.2, hNav1.7, hNav1.4, and rNav1.5) with seven AlphaFold2 structures (hNav2.1, hNav1.4, hNav1.5, rNav1.5, mNav1.5, hNav1.4, and hNav1.9) shows similar folding and backbone conformations (Figure 4A,B). Structural deviations between the AlphaFold2 and experimental structures, on one hand, and between different experimental structures, on the other hand, are similar. A more detailed comparison is provided for Kv channels. Figure $4 \mathrm{C}$ shows the superimposed crystal structure of the Kv1.2-Kv2.1 channel with the AlphaFold2 structures of the voltage-gated potassium channels hKv1.2, hKv1.6, hKv2.1, and hKv3.1. The backbone conformations, positions, and orientation of the CA-CB bonds in these structures are very similar. RMS deviations $(\AA)$ of alpha carbons in the membrane segments of the Kv1.2 channel are as small as follows: S1, 1.2; S2, 1.1; S3, 1.5; S4, 0.73; S5, 0.46; P, 0.24; and S6, 0.53. The RMSD value for the P-helix is the smallest one because this segment was used for the 3D alignment. Thus, all details, including the open-gate conformation of the S6 bundle, are precisely predicted in the AlphaFold 2 model. The 
AlphaFold2 models for the hKv1.6, hKv2.1, and hKv3.1 channels are also very close to the experimental structures.

A

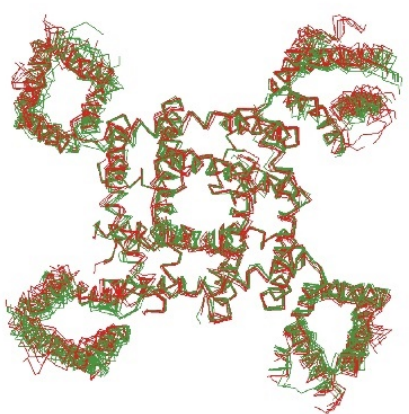

VSD

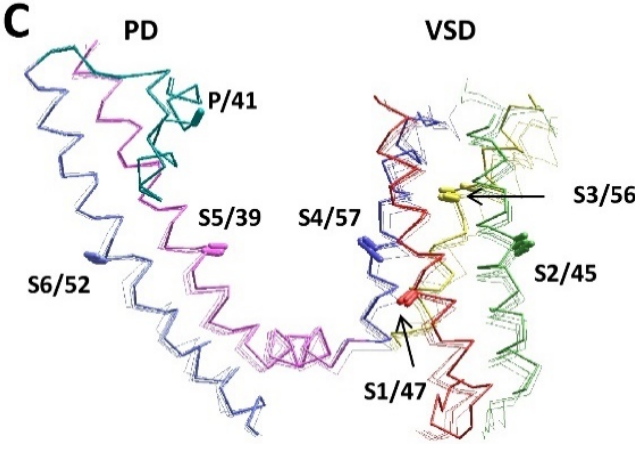

B

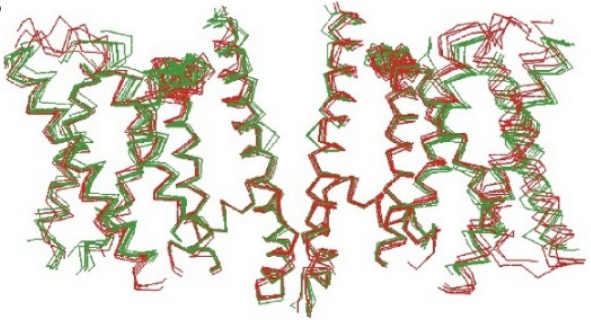

Figure 4. Similarity of experimental and AlphaFold2 structures of voltage-gated potassium and sodium channels. (A,B) Intracellular (A) and intra-membrane (B) views of the experimental (green) and AlphaFold2 (red) structures of voltage-gated sodium channels. Shown are the experimental structures of hNav1.2 (6j8e), hNav1.7 (6j8j), hNav1.4 (6agf), and rNav1.5 (6uz3), and the AlphaFold2 structures of hNav2.1 (Q01118), hNav1.4 (P35499), hNav1.5 (Q14524), rNav1.5 (P15389), mNav1.5 (Q9JJV9), hNav1.4 (P35499), and hNav1.9 (Q9UI33). For clarity, only repeats II and IV are shown in (B,C). (C) Crystal structure of the Kv1.2 channel (DPB ID: 2R9R) superimposed with the AlphaFold2 structures of hKv1.2 (P16389), hKv1.6 (P17658), hKv2.1 (Q14721), and hKv3.1 (P48547). Helices S1, S2, S3, S4, S5, and S6 are red, green, yellow, blue, magenta, and violet, respectively. P-loops are cyan. The CA-CB bonds of residues in matching positions are shown by sticks and labeled as in Figure 1D. The AlphaFold2 and experimental structures are very similar.

Figure 5A shows the experimental structures and AlphaFold2 models of two potassium channels: Kv1.2 (2R9R vs. P16389) and Kv7.1 (6uzz vs. P51787). Folding of the P-loops in the two channels is similar, but the folding of VSDs is rather different. The activation gate conformations (C-part of S6 and N-part of S5) also differ significantly. It is interesting, however, that the AlphaFold2 and experimental structures of each channel are similar.

The next example includes VSD-IV of the hNav1.5 (Q14524) and hNav2.1 (Q01118) channels, where the CA-CB bonds of residues in sequentially matching positions of helices IVS1, IVS2 and IVS3 have similar orientations. However, there is a one-helical shift of helix IVS4 in the Nav2.1 channel towards the cytoplasm due to an additional helical turn between helix IVS4 and the linker-helix IVS4-S5 (Figure 5B). Since Nav2.1 (SCN7A) is not a voltage-gated channel, the atypical conformation of the $\mathrm{S} 4$ helix is not surprising. We are not aware of experimental structures of the Nav2.1 channel or other structures with atypical folding of the $S 4$ helix.

Figure 5 C shows an example of differences between P-loop channels. Although the P1 helices are the most structurally conserved elements of P-loop channels, the experimental structures demonstrate subtle, but notable differences between potassium and potassiumlike glutamate receptor channels, on one hand, and sodium and calcium channels, on the other hand. In the latter family, the P-helices are about a half-turn more distant from the 
pore axis. The AlphaFold 2 models readily reproduced this difference. Within each family, the pore helices of experimental and modeled structures are hardly distinguishable.

A
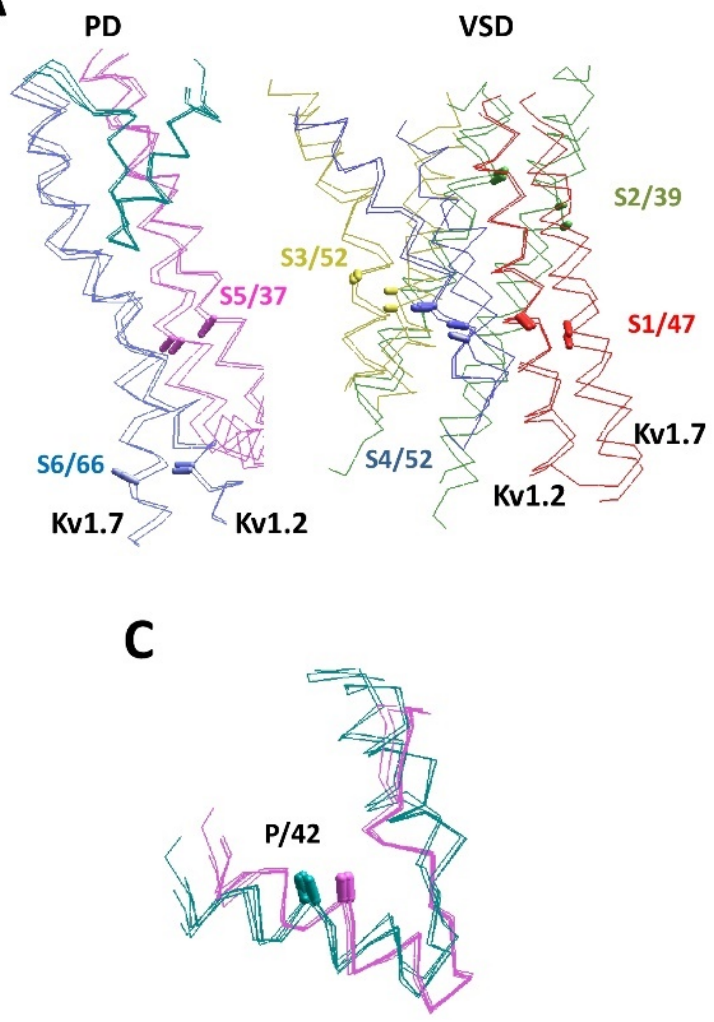

B

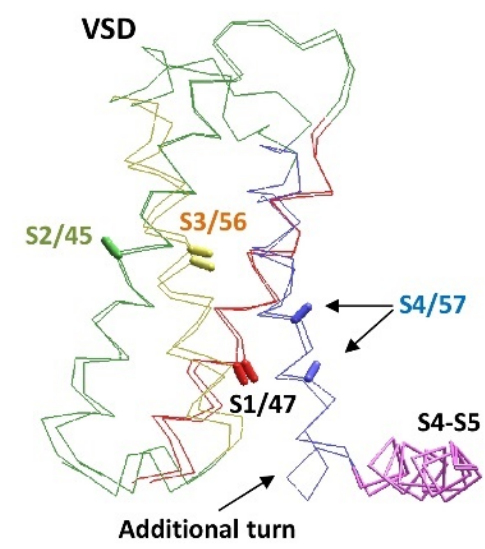

Figure 5. Structural peculiarities of voltage-gated P-loop channels. (A) The superimposition of experimental and AlphaFold2 structures of the Kv1.2 (2R9R and P16389) and Kv7.1 (6uzz and P51787) channels. Helices S1, S2, S3, S4, S5, and S6 are red, green, yellow, blue, magenta, and violet, respectively. P-loops are cyan. The CA-CB bonds of residues in matching positions are shown by sticks and labeled as in Figure 1D. Structures Kv1.2 and Kv7.1 are significantly different, but the AlphaFold2 and experimental structures of each channel are similar. (B) VSD-IV in channels hNav1.5 (Q14524) and hNav2.1 (Q01118). The CA-CB bonds of homologous residues in transmembrane segments match well, except for helix IVS4. In Nav2.1, the helix is shifted towards the cytoplasm due to the appearance of an additional helical turn between helix IVS4 and linker-helix S4-S5. (C) P-loops in the experimental and AlphaFold2 structures of potassium channels (cyan) are significantly different from sodium and calcium channels (magenta), but within each subfamily, the experimental and AlphaFold2 structures are similar. Shown are channels Kv1.2-2.1 (2r9r), hKv1.5 (6uzz), rbCav1.1 (5gjv), hNav1.4 (6agf), hKv7.1 (P51787), hKv2.1 (Q14721), hKv1.2 (P16389), hKv1.6 (P17658), hKv3.1 (P48547), hCav3.1 (O43497), hNav1.2 (Q99250), and hNav1.5 (Q14524).

The majority of channels with voltage-sensing domains have so-called swappeddomain architecture with a given VSD approaching the neighboring-domain quarter of the pore module (Figure 1C). However, non-swapped architecture is seen in some channels, e.g., hERG, $\mathrm{K}_{\mathrm{Ca}} 1.1$, and TRPV6 (5iwk). An alternative structure of the TRPV6 channel with resolved S4-S5 linkers (6e2f) shows the classical domain-swapping organization. AlphaFold2 structures of the $\mathrm{K}_{\mathrm{Ca}} 1.1$ and hERG channels have the non-swap domain architecture, whereas TRPV6 and other TRP channels have the swapped-domain architecture.

We further considered some sodium, calcium, and TRP channels with distortions caused by $\pi$-bulges. These channels have conserved asparagine residues in positions $\mathrm{S} 6 / 56$ (Figure 1D), which likely appeared in evolution as insertions [139] and induced $\pi$-bulges to preserve residue orientations in the S6 helices. The bulges are usually formed one 
turn upstream of the conserved asparagine, whose sidechain can donate an H-bond to the "bachelor" backbone carbonyl, and thus stabilize the bulge. The difference between S6 conformations in structures with and without $\pi$-bulges is shown in Figure 6A. The CA-CB bonds of residues in positions S6/46 and S6/56 are shown as sticks. Positions and orientations of $\mathrm{CA}-\mathrm{CB}$ bonds in residues $\mathrm{S} 6 / 46$ are well conserved. However, $\mathrm{CA}-\mathrm{CB}$ bonds of residues $\mathrm{S} 6 / 56$ are split due to the $\pi$-helix bulge at position S6/51 in TRPA1 (O75762) and TRPV3 (6lgp). AlphaFold2 structures of TRPM2 (O94759), TRPA1 (O75762), TRPV6 (Q9H1D0), TRPV1 (Q8NER1), and TRPV3 (Q8NET8) also have a $\pi$-bulge in S6.

A

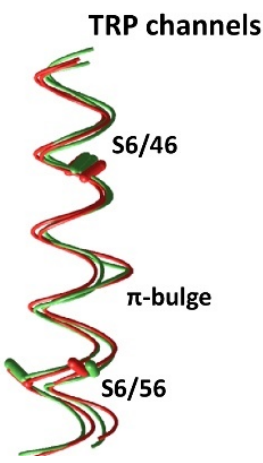

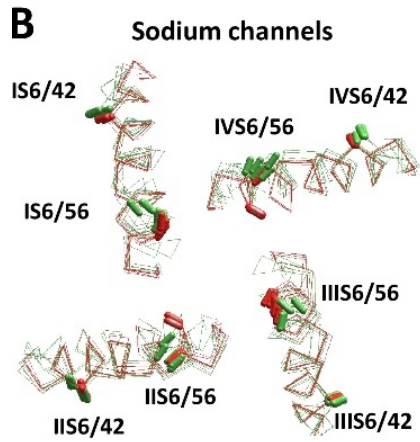

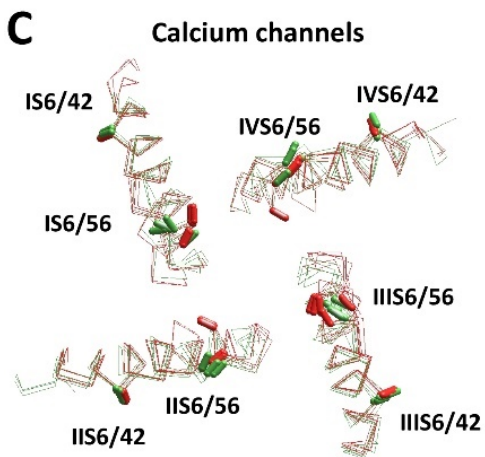

Figure 6. Diverse conformations of S6 helices in experimental and AlphaFold2 structures. (A) S6 helices in TRP channels. Experimental structures of TRPV6 (5iwk) and TRPV3 (6lgp) are green. AlphaFold2 structures of TRPV2 (Q9Y5S1) and TRPA1 (O75762) are red. Positions and orientations of the $\mathrm{CA}-\mathrm{CB}$ bonds of residues $\mathrm{S} 6 / 46$ are well conserved. In contrast, the CA-CB bonds of residues S6/56 are split due to the $\pi$-helix bulge at position S6/51 in the TRPA1 (O75762) and TRPV3 (6lgp) structures. (B,C) S6 helices in eukaryotic sodium (B) and calcium (C) channels. Experimental and AlphaFold2 structures are green and red, respectively. (B) Sodium channels hNav1.2 (6j8e), hNav1.7 (6j8j, 6n4q), hNav1.4 (6agf/P35499), rNav1.5 (6uz3/P15389), hNav2.1 (Q01118), hNav1.5 (Q14524), mNav1.5 (Q9JJV9), and hNav1.9 (Q9UI33). (C) Calcium channels hCav3.1 (6kzo/O43497), rbCav1.1 (6jpa, 6jp5, 6byo and 5gjv), hCav1.1 (Q13698), hCav1.4 (O60840), hCav1.3 (Q01668), and hCav3.2 (O95180). The CA-CB bonds of residues S6/42 and S6/65 (Figure 1D) are shown by sticks. The positions and orientations of the CA-CB bonds in position S6/42 are similar in the experimental and AlphaFold 2 structures. However, due to the different patterns of $\pi$-helical bulges, the orientation of asparagines in position S6/56 varies between repeats of individual channels and between different structures.

Figure $6 \mathrm{~B}, \mathrm{C}$ shows intracellular views of the $\mathrm{S} 6$ bundle in eight sodium channels and six calcium channels. Folding of the transmembrane segments in the AlphaFold 2 models is very similar to that in respective experimental structures. Particularly, residues in position S6/42 are well conserved in both experimental and AlphaFold2 structures. In contrast, residues in position $\mathrm{S6} / 56$ are split in two groups, depending on the presence or absence of $\pi$-bulges. The orientation of asparagines in position $S 6 / 56$ varies between individual channels, between repeats of these channels, and between experimental structures and AlphaFold2 models.

The experimental structures of the Nav1.2, Nav1.4, Nav1.5, and Nav1.7 channels have bulges in IS6 and IIIS6. Most of the AlphaFold2 models of channels hNav1.1, hNav1.2, hNav1.4, hNav1.5, and hNav1.9 have $\pi$-bulges in helices IS6, IIIS6, and IVS6, whereas the experimental structures lack a $\pi$-bulge in IVS6. AlphaFold2 models of the mNav1.5 and hNav1.9 channels have $\pi$-bulges in all of the four repeats, which match the $\pi$-bulge pattern in the experimental structure of NavPaS. Interestingly, the AlphaFold2 model of mNav1.5 differs from the rNv1.5 and hNav1.5 models, despite the sequences of these channels being very similar. Bulges in IS6 and IIIS6 are predicted for Nav2.1, which is not a voltage-gated channel. 
A similar situation is observed in calcium channels. The available experimental structures of these channels are split in several classes according to the presence or absence of $\pi$-helix bulges in the S6 helices [30]. The Class I structure has no $\pi$-bulges and Class II has $\pi$-bulges in repeats I, II, and III, whereas the Class III structure has bulges in repeats I and II. The AlphaFold2 model of the T-type calcium channel hCav3.1 reproduced $\pi$-bulges, which are seen in the experimental Class II structure. The AlphaFold2 model of Cav1.1 has a $\pi$-bulge only in repeat I. AlphaFold2 models of Cav1.3, Cav1.4, Cav2.1, and Cav2.2 have bulges in repeats I and III, which are often seen in experimental structures of sodium, but not calcium channels. The AlphaFold2 models of hCav3.2 and hCav3.3 have $\pi$-bulges in all four repeats.

Thus, patterns of $\pi$-helix bulges in the $\mathrm{S} 6$ helices are highly diverse in both the experimental structures and the AlphaFold 2 models. The causes of the differences are unknown. Likely, there are alternative conformations with similar energies, and therefore transitions between the conformations are possible. Structural determinants underlying such transitions are unknown. This is an intriguing problem in the field of the structural biology of P-loop channels.

The AlphaFold2 publications mention a limited reliability of membrane protein models. However, the above comparison of the AlphaFold2 models and experimental structures of P-loop channels shows an impressive predictive power of the artificial neural network in this particular class of important membrane proteins.

\section{Perspectives}

Further studies of P-loop channels in different states and in complexes with different ligands are necessary to address challenging problems involving the mechanisms of disease mutations. Since AlphaFold2 does not necessarily predict the consequences of missense mutations [148], such studies should involve a combination of experimental and theoretical approaches. P-loop channels are regulated by various auxiliary subunits and multiple cytoplasmic proteins, e.g., [149-152]. Some cryo-EM structures show complexes of channels with auxiliary subunits. However, the 3D structures of large cytoplasmic parts of many P-loop channels, which are targeted by various proteins, are not resolved in either cryo-EM structures or AlphFold2 models. Predicting the structures of P-loop channels with cytoplasmic proteins is of paramount importance for understanding the mechanisms by which disease mutations of the cytoplasmic proteins cause the ion channel dysfunction. Mutational studies, which reveal residues involved in protein-protein interactions, may provide important constraints to predict the protein-protein complexes using neural networks or physics-based protein-protein docking software. Another major problem is that the vast majority of experimental structures show P-loop channels in the energetically most-preferable states and neural-network software, which is trained on these structures, also predicts energetically preferable structures. Computational approaches may be used to transfer the energetically preferable experimental structures to low-populated, but functionally important states. Such models help to understand the mechanisms of disease mutations and ligand action [95,96,153]. Computational studies including molecular dynamic simulations and high-throughput ligands docking will benefit from the available experimental structures and neural-network-based models of P-loop channels.

Funding: This research received no external funding.

Institutional Review Board Statement: Not applicable.

Informed Consent Statement: Not applicable.

Data Availability Statement: Not applicable.

Conflicts of Interest: The authors declare no conflict of interest. 


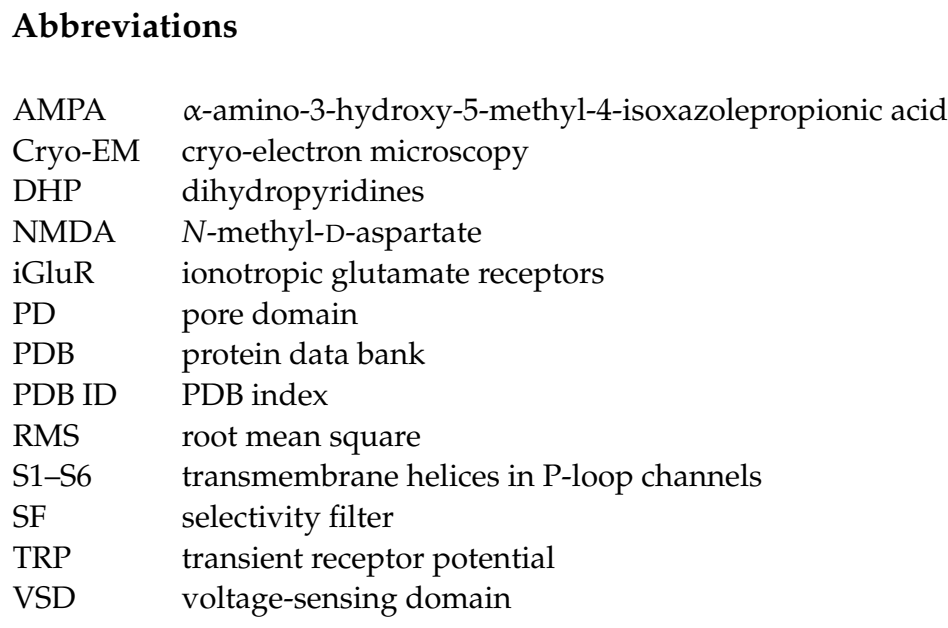

\section{References}

1. Catterall, W.A. Voltage-gated calcium channels. Cold Spring Harb. Perspect. Biol. 2011, 3, a003947. [CrossRef] [PubMed]

2. Catterall, W.A. Voltage-gated sodium channels at 60: Structure, function and pathophysiology. J. Physiol. 2012, 590, 2577-2589. [CrossRef] [PubMed]

3. Wulff, H.; Zhorov, B.S. $\mathrm{K}^{+}$channel modulators for the treatment of neurological disorders and autoimmune diseases. Chem. Rev. 2008, 108, 1744-1773. [CrossRef]

4. MacKinnon, R. Potassium channels. FEBS Lett. 2003, 555, 62-65. [CrossRef]

5. Venkatachalam, K.; Montell, C. TRP channels. Annu. Rev. Biochem. 2007, 76, 387-417. [CrossRef]

6. Traynelis, S.F.; Wollmuth, L.P.; McBain, C.J.; Menniti, F.S.; Vance, K.M.; Ogden, K.K.; Hansen, K.B.; Yuan, H.; Myers, S.J.; Dingledine, R. Glutamate receptor ion channels: Structure, regulation, and function. Pharmacol. Rev. 2010, 62, 405-496. [CrossRef]

7. Hansen, K.B.; Wollmuth, L.P.; Bowie, D.; Furukawa, H.; Menniti, F.S.; Sobolevsky, A.I.; Swanson, G.T.; Swanger, S.A.; Greger, I.H.; Nakagawa, T.; et al. Structure, Function, and Pharmacology of Glutamate Receptor Ion Channels. Pharmacol. Rev. 2021, 73, 298-487. [CrossRef]

8. Noreng, S.; Li, T.; Payandeh, J. Structural Pharmacology of Voltage-Gated Sodium Channels. J. Mol. Biol. 2021, $433,166967$. [CrossRef]

9. Catterall, W.A.; Lenaeus, M.J.; Gamal El-Din, T.M. Structure and Pharmacology of Voltage-Gated Sodium and Calcium Channels. Annu. Rev. Pharmacol. Toxicol. 2020, 60, 133-154. [CrossRef]

10. Huang, Y.; Fliegert, R.; Guse, A.H.; Lu, W.; Du, J. A structural overview of the ion channels of the TRPM family. Cell Calcium 2020, 85, 102111. [CrossRef]

11. Mayer, M.L.; Armstrong, N. Structure and function of glutamate receptor ion channels. Annu. Rev. Physiol. 2004, 66, 161-181. [CrossRef] [PubMed]

12. Liu, C.; Montell, C. Forcing open TRP channels: Mechanical gating as a unifying activation mechanism. Biochem. Biophys. Res. Commun. 2015, 460, 22-25. [CrossRef] [PubMed]

13. Grizel, A.V.; Glukhov, G.S.; Sokolova, O.S. Mechanisms of activation of voltage-gated potassium channels. Acta Nat. 2014, 6, 10-26. [CrossRef]

14. Bagneris, C.; Naylor, C.E.; McCusker, E.C.; Wallace, B.A. Structural model of the open-closed-inactivated cycle of prokaryotic voltage-gated sodium channels. J. Gen. Physiol. 2015, 145, 5-16. [CrossRef]

15. Lipscombe, D.; Helton, T.D.; Xu, W. L-type calcium channels: The low down. J. Neurophysiol. 2004, 92, 2633-2641. [CrossRef]

16. Mazzolini, M.; Marchesi, A.; Giorgetti, A.; Torre, V. Gating in CNGA1 channels. Pflug. Arch. 2010, 459, 547-555. [CrossRef]

17. Enyedi, P.; Czirjak, G. Molecular background of leak $\mathrm{K}^{+}$currents: Two-pore domain potassium channels. Physiol. Rev. 2010, 90, 559-605. [CrossRef]

18. Zhorov, B.S.; Tikhonov, D.B. Potassium, sodium, calcium and glutamate-gated channels: Pore architecture and ligand action. J. Neurochem. 2004, 88, 782-799. [CrossRef]

19. Wollmuth, L.P.; Sobolevsky, A.I. Structure and gating of the glutamate receptor ion channel. Trends Neurosci. 2004, 27, 321-328. [CrossRef]

20. Armstrong, C.M.; Hille, B. The inner quaternary ammonium ion receptor in potassium channels of the node of Ranvier. J. Gen. Physiol. 1972, 59, 388-400. [CrossRef]

21. Heinemann, S.H.; Terlau, H.; Stuhmer, W.; Imoto, K.; Numa, S. Calcium channel characteristics conferred on the sodium channel by single mutations. Nature 1992, 356, 441-443. [CrossRef] [PubMed]

22. Dudley, S.C., Jr.; Chang, N.; Hall, J.; Lipkind, G.; Fozzard, H.A.; French, R.J. $\mu$-conotoxin GIIIA interactions with the voltage-gated $\mathrm{Na}^{+}$channel predict a clockwise arrangement of the domains. J. Gen. Physiol. 2000, 116, 679-690. [CrossRef] [PubMed]

23. Korkosh, V.S.; Zhorov, B.S.; Tikhonov, D.B. Analysis of inter-residue contacts reveals folding stabilizers in P-loops of potassium, sodium, and TRPV channels. Eur. Biophys. J. 2016, 45, 321-329. [CrossRef] [PubMed] 
24. Kim, D.M.; Nimigean, C.M. Voltage-Gated Potassium Channels: A Structural Examination of Selectivity and Gating. Cold Spring Harb. Perspect. Biol. 2016, 8, a029231. [CrossRef]

25. Varadi, G.; Strobeck, M.; Koch, S.; Caglioti, L.; Zucchi, C.; Palyi, G. Molecular elements of ion permeation and selectivity within calcium channels. Crit. Rev. Biochem. Mol. Biol. 1999, 34, 181-214. [CrossRef]

26. Doyle, D.A.; Morais Cabral, J.; Pfuetzner, R.A.; Kuo, A.; Gulbis, J.M.; Cohen, S.L.; Chait, B.T.; MacKinnon, R. The structure of the potassium channel: Molecular basis of $\mathrm{K}^{+}$conduction and selectivity. Science 1998, 280, 69-77. [CrossRef]

27. Sievers, F.; Wilm, A.; Dineen, D.; Gibson, T.J.; Karplus, K.; Li, W.; Lopez, R.; McWilliam, H.; Remmert, M.; Soding, J.; et al. Fast, scalable generation of high-quality protein multiple sequence alignments using Clustal Omega. Mol. Syst. Biol. 2011, 7, 539. [CrossRef]

28. Long, S.B.; Tao, X.; Campbell, E.B.; MacKinnon, R. Atomic structure of a voltage-dependent $\mathrm{K}^{+}$channel in a lipid membrane-like environment. Nature 2007, 450, 376-382. [CrossRef]

29. Tikhonov, D.B.; Zhorov, B.S. Architecture and pore block of eukaryotic voltage-gated sodium channels in view of NavAb bacterial sodium channel structure. Mol. Pharmacol. 2012, 82, 97-104. [CrossRef]

30. Wu, J.; Yan, Z.; Li, Z.; Qian, X.; Lu, S.; Dong, M.; Zhou, Q.; Yan, N. Structure of the voltage-gated calcium channel Ca(v)1.1 at 3.6 A resolution. Nature 2016, 537, 191-196. [CrossRef]

31. Fan, C.; Sukomon, N.; Flood, E.; Rheinberger, J.; Allen, T.W.; Nimigean, C.M. Ball-and-chain inactivation in a calcium-gated potassium channel. Nature 2020, 580, 288-293. [CrossRef]

32. Zhao, Y.; Huang, G.; Wu, J.; Wu, Q.; Gao, S.; Yan, Z.; Lei, J.; Yan, N. Molecular Basis for Ligand Modulation of a Mammalian Voltage-Gated $\mathrm{Ca}^{2+}$ Channel. Cell 2019, 177, 1495-1506.e12. [CrossRef]

33. Clayton, G.M.; Altieri, S.; Heginbotham, L.; Unger, V.M.; Morais-Cabral, J.H. Structure of the transmembrane regions of a bacterial cyclic nucleotide-regulated channel. Proc. Natl. Acad. Sci. USA 2008, 105, 1511-1515. [CrossRef]

34. Gao, S.; Yan, N. Structural Basis of the Modulation of the Voltage-Gated Calcium Ion Channel Cav 1.1 by Dihydropyridine Compounds. Angew. Chem. Int. Ed. Engl. 2021, 60, 3131-3137. [CrossRef]

35. Sun, J.; MacKinnon, R. Structural Basis of Human KCNQ1 Modulation and Gating. Cell 2020, 180, 340-347.e9. [CrossRef]

36. Zhao, Y.; Huang, G.; Wu, Q.; Wu, K.; Li, R.; Lei, J.; Pan, X.; Yan, N. Cryo-EM structures of apo and antagonist-bound human Cav3.1. Nature 2019, 576, 492-497. [CrossRef]

37. Wang, W.; MacKinnon, R. Cryo-EM Structure of the Open Human Ether-a-go-go-Related K+ Channel hERG. Cell 2017, 169, 422-430.e10. [CrossRef]

38. Whorton, M.R.; MacKinnon, R. X-ray structure of the mammalian GIRK2-betagamma G-protein complex. Nature 2013, 498, 190-197. [CrossRef]

39. Kong, C.; Zeng, W.; Ye, S.; Chen, L.; Sauer, D.B.; Lam, Y.; Derebe, M.G.; Jiang, Y. Distinct gating mechanisms revealed by the structures of a multi-ligand gated $\mathrm{K}^{+}$channel. Elife 2012, 1, e00184. [CrossRef]

40. Tao, X.; Hite, R.K.; MacKinnon, R. Cryo-EM structure of the open high-conductance $\mathrm{Ca}^{2+}$-activated $\mathrm{K}^{+}$channel. Nature 2017, 541, 46-51. [CrossRef] [PubMed]

41. Payandeh, J.; Scheuer, T.; Zheng, N.; Catterall, W.A. The crystal structure of a voltage-gated sodium channel. Nature 2011, 475, 353-358. [CrossRef]

42. Twomey, E.C.; Yelshanskaya, M.V.; Vassilevski, A.A.; Sobolevsky, A.I. Mechanisms of Channel Block in Calcium-Permeable AMPA Receptors. Neuron 2018, 99, 956-968.e4. [CrossRef]

43. Lenaeus, M.J.; Gamal El-Din, T.M.; Ing, C.; Ramanadane, K.; Pomes, R.; Zheng, N.; Catterall, W.A. Structures of closed and open states of a voltage-gated sodium channel. Proc. Natl. Acad. Sci. USA 2017, 114, E3051-E3060. [CrossRef] [PubMed]

44. Regan, M.C.; Grant, T.; McDaniel, M.J.; Karakas, E.; Zhang, J.; Traynelis, S.F.; Grigorieff, N.; Furukawa, H. Structural Mechanism of Functional Modulation by Gene Splicing in NMDA Receptors. Neuron 2018, 98, 521-529.e3. [CrossRef]

45. Wisedchaisri, G.; Tonggu, L.; McCord, E.; Gamal El-Din, T.M.; Wang, L.; Zheng, N.; Catterall, W.A. Resting-State Structure and Gating Mechanism of a Voltage-Gated Sodium Channel. Cell 2019, 178, 993-1003.e12. [CrossRef]

46. Paulsen, C.E.; Armache, J.P.; Gao, Y.; Cheng, Y.; Julius, D. Structure of the TRPA1 ion channel suggests regulatory mechanisms. Nature 2015, 520, 511-517. [CrossRef]

47. McCusker, E.C.; Bagneris, C.; Naylor, C.E.; Cole, A.R.; D'Avanzo, N.; Nichols, C.G.; Wallace, B.A. Structure of a bacterial voltage-gated sodium channel pore reveals mechanisms of opening and closing. Nat. Commun. 2012, 3, 1102. [CrossRef]

48. Cao, E.; Liao, M.; Cheng, Y.; Julius, D. TRPV1 structures in distinct conformations reveal activation mechanisms. Nature 2013, 504, 113-118. [CrossRef] [PubMed]

49. Zhang, X.; Ren, W.; DeCaen, P.; Yan, C.; Tao, X.; Tang, L.; Wang, J.; Hasegawa, K.; Kumasaka, T.; He, J.; et al. Crystal structure of an orthologue of the NaChBac voltage-gated sodium channel. Nature 2012, 486, 130-134. [CrossRef]

50. Zubcevic, L.; Herzik, M.A., Jr.; Chung, B.C.; Liu, Z.; Lander, G.C.; Lee, S.Y. Cryo-electron microscopy structure of the TRPV2 ion channel. Nat. Struct. Mol. Biol. 2016, 23, 180-186. [CrossRef] [PubMed]

51. Shen, H.; Li, Z.; Jiang, Y.; Pan, X.; Wu, J.; Cristofori-Armstrong, B.; Smith, J.J.; Chin, Y.K.Y.; Lei, J.; Zhou, Q.; et al. Structural basis for the modulation of voltage-gated sodium channels by animal toxins. Science 2018, 362. [CrossRef] [PubMed]

52. Singh, A.K.; McGoldrick, L.L.; Sobolevsky, A.I. Structure and gating mechanism of the transient receptor potential channel TRPV3. Nat. Struct. Mol. Biol. 2018, 25, 805-813. [CrossRef] [PubMed] 
53. Pan, X.; Li, Z.; Huang, X.; Huang, G.; Gao, S.; Shen, H.; Liu, L.; Lei, J.; Yan, N. Molecular basis for pore blockade of human Na ${ }^{+}$ channel $\mathrm{Na}_{\mathrm{v}} 1.2$ by the $\mu$-conotoxin KIIIA. Science 2019, 363, 1309-1313. [CrossRef]

54. Zubcevic, L.; Herzik, M.A., Jr.; Wu, M.; Borschel, W.F.; Hirschi, M.; Song, A.S.; Lander, G.C.; Lee, S.Y. Conformational ensemble of the human TRPV3 ion channel. Nat. Commun. 2018, 9, 4773. [CrossRef]

55. Pan, X.; Li, Z.; Zhou, Q.; Shen, H.; Wu, K.; Huang, X.; Chen, J.; Zhang, J.; Zhu, X.; Lei, J.; et al. Structure of the human voltage-gated sodium channel Nav1.4 in complex with beta1. Science 2018, 362, eaau2486. [CrossRef]

56. Yan, Z.; Zhou, Q.; Wang, L.; Wu, J.; Zhao, Y.; Huang, G.; Peng, W.; Shen, H.; Lei, J.; Yan, N. Structure of the Nav1.4-beta1 Complex from Electric Eel. Cell 2017, 170, 470-482.e11. [CrossRef]

57. Singh, A.K.; McGoldrick, L.L.; Demirkhanyan, L.; Leslie, M.; Zakharian, E.; Sobolevsky, A.I. Structural basis of temperature sensation by the TRP channel TRPV3. Nat. Struct. Mol. Biol. 2019, 26, 994-998. [CrossRef]

58. Jiang, D.; Shi, H.; Tonggu, L.; Gamal El-Din, T.M.; Lenaeus, M.J.; Zhao, Y.; Yoshioka, C.; Zheng, N.; Catterall, W.A. Structure of the Cardiac Sodium Channel. Cell 2020, 180, 122-134.e10. [CrossRef]

59. Jiang, D.; Banh, R.; Gamal El-Din, T.M.; Tonggu, L.; Lenaeus, M.J.; Pomes, R.; Zheng, N.; Catterall, W.A. Open-state structure and pore gating mechanism of the cardiac sodium channel. Cell 2021, 184, 5151-5162.e11. [CrossRef]

60. Deng, Z.; Maksaev, G.; Rau, M.; Xie, Z.; Hu, H.; Fitzpatrick, J.A.J.; Yuan, P. Gating of human TRPV3 in a lipid bilayer. Nat. Struct. Mol. Biol. 2020, 27, 635-644. [CrossRef]

61. Jiang, D.; Tonggu, L.; Gamal El-Din, T.M.; Banh, R.; Pomes, R.; Zheng, N.; Catterall, W.A. Structural basis for voltage-sensor trapping of the cardiac sodium channel by a deathstalker scorpion toxin. Nat. Commun. 2021, 12, 128. [CrossRef]

62. Shimada, H.; Kusakizako, T.; Dung Nguyen, T.H.; Nishizawa, T.; Hino, T.; Tominaga, M.; Nureki, O. The structure of lipid nanodisc-reconstituted TRPV3 reveals the gating mechanism. Nat. Struct. Mol. Biol. 2020, 27, 645-652. [CrossRef]

63. Li, Z.; Jin, X.; Wu, T.; Huang, G.; Wu, K.; Lei, J.; Pan, X.; Yan, N. Structural Basis for Pore Blockade of the Human Cardiac Sodium Channel Nav 1.5 by the Antiarrhythmic Drug Quinidine. Angew. Chem. Int. Ed. Engl. 2021, 60, 11474-11480. [CrossRef]

64. Shen, H.; Liu, D.; Wu, K.; Lei, J.; Yan, N. Structures of human Nav1.7 channel in complex with auxiliary subunits and animal toxins. Science 2019, 363, 1303-1308. [CrossRef]

65. Xu, H.; Li, T.; Rohou, A.; Arthur, C.P.; Tzakoniati, F.; Wong, E.; Estevez, A.; Kugel, C.; Franke, Y.; Chen, J.; et al. Structural Basis of Nav1.7 Inhibition by a Gating-Modifier Spider Toxin. Cell 2019, 176, 1238-1239. [CrossRef]

66. Saotome, K.; Singh, A.K.; Yelshanskaya, M.V.; Sobolevsky, A.I. Crystal structure of the epithelial calcium channel TRPV6. Nature 2016, 534, 506-511. [CrossRef]

67. Singh, A.K.; McGoldrick, L.L.; Twomey, E.C.; Sobolevsky, A.I. Mechanism of calmodulin inactivation of the calcium-selective TRP channel TRPV6. Sci. Adv. 2018, 4, eaau6088. [CrossRef]

68. Wang, L.; Fu, T.M.; Zhou, Y.; Xia, S.; Greka, A.; Wu, H. Structures and gating mechanism of human TRPM2. Science 2018, 362, eaav4809. [CrossRef]

69. She, J.; Guo, J.; Chen, Q.; Zeng, W.; Jiang, Y.; Bai, X.C. Structural insights into the voltage and phospholipid activation of the mammalian TPC1 channel. Nature 2018, 556, 130-134. [CrossRef]

70. McGoldrick, L.L.; Singh, A.K.; Saotome, K.; Yelshanskaya, M.V.; Twomey, E.C.; Grassucci, R.A.; Sobolevsky, A.I. Opening of the human epithelial calcium channel TRPV6. Nature 2018, 553, 233-237. [CrossRef]

71. Jumper, J.; Evans, R.; Pritzel, A.; Green, T.; Figurnov, M.; Ronneberger, O.; Tunyasuvunakool, K.; Bates, R.; Zidek, A.; Potapenko, A.; et al. Highly accurate protein structure prediction with AlphaFold. Nature 2021, 596, 583-589. [CrossRef]

72. Varadi, M.; Anyango, S.; Deshpande, M.; Nair, S.; Natassia, C.; Yordanova, G.; Yuan, D.; Stroe, O.; Wood, G.; Laydon, A.; et al AlphaFold Protein Structure Database: Massively expanding the structural coverage of protein-sequence space with high-accuracy models. Nucleic Acids Res. 2022, 50, D439-D444. [CrossRef]

73. Tikhonov, D.B.; Zhorov, B.S.; Magazanik, L.G. Intersegment hydrogen bonds as possible structural determinants of the N/Q/R site in glutamate receptors. Biophys. J. 1999, 77, 1914-1926. [CrossRef]

74. Lipkind, G.M.; Fozzard, H.A. Molecular modeling of interactions of dihydropyridines and phenylalkylamines with the inner pore of the L-type $\mathrm{Ca}^{2+}$ channel. Mol. Pharmacol. 2003, 63, 499-511. [CrossRef]

75. Lipkind, G.M.; Fozzard, H.A. Molecular modeling of local anesthetic drug binding by voltage-gated sodium channels. Mol. Pharmacol. 2005, 68, 1611-1622. [CrossRef]

76. Tikhonov, D.B.; Zhorov, B.S. Modeling P-loops domain of sodium channel: Homology with potassium channels and interaction with ligands. Biophys. J. 2005, 88, 184-197. [CrossRef]

77. O'Reilly, A.O.; Khambay, B.P.; Williamson, M.S.; Field, L.M.; Wallace, B.A.; Davies, T.G. Modelling insecticide-binding sites in the voltage-gated sodium channel. Biochem. J. 2006, 396, 255-263. [CrossRef]

78. Huber, I.; Wappl, E.; Herzog, A.; Mitterdorfer, J.; Glossmann, H.; Langer, T.; Striessnig, J. Conserved Ca2+-antagonist-binding properties and putative folding structure of a recombinant high-affinity dihydropyridine-binding domain. Biochem. J. 2000, 347 Pt 3, 829-836. [CrossRef]

79. Corry, B.; Vora, T.; Chung, S.H. Electrostatic basis of valence selectivity in cationic channels. Biochim. Biophys. Acta 2005, 1711, 72-86. [CrossRef]

80. Cosconati, S.; Marinelli, L.; Lavecchia, A.; Novellino, E. Characterizing the 1,4-dihydropyridines binding interactions in the L-type $\mathrm{Ca}^{2+}$ channel: Model construction and docking calculations. J. Med. Chem. 2007, 50, 1504-1513. [CrossRef]

81. Catterall, W.A.; Striessnig, J. Receptor sites for $\mathrm{Ca}^{2+}$ channel antagonists. Trends Pharmacol. Sci. 1992, 13, 256-262. [CrossRef] 
82. Hockerman, G.H.; Peterson, B.Z.; Johnson, B.D.; Catterall, W.A. Molecular determinants of drug binding and action on L-type calcium channels. Annu. Rev. Pharmacol. Toxicol. 1997, 37, 361-396. [CrossRef]

83. Alpert, L.A.; Fozzard, H.A.; Hanck, D.A.; Makielski, J.C. Is there a second external lidocaine binding site on mammalian cardiac cells? Am. J. Physiol. 1989, 257, H79-H84. [CrossRef]

84. Jiang, Y.; Lee, A.; Chen, J.; Cadene, M.; Chait, B.T.; MacKinnon, R. Crystal structure and mechanism of a calcium-gated potassium channel. Nature 2002, 417, 515-522. [CrossRef]

85. Long, S.B.; Campbell, E.B.; Mackinnon, R. Crystal structure of a mammalian voltage-dependent Shaker family K ${ }^{+}$channel. Science 2005, 309, 897-903. [CrossRef]

86. Cordero-Morales, J.F.; Cuello, L.G.; Zhao, Y.; Jogini, V.; Cortes, D.M.; Roux, B.; Perozo, E. Molecular determinants of gating at the potassium-channel selectivity filter. Nat. Struct. Mol. Biol. 2006, 13, 311-318. [CrossRef]

87. Cuello, L.G.; Jogini, V.; Cortes, D.M.; Pan, A.C.; Gagnon, D.G.; Dalmas, O.; Cordero-Morales, J.F.; Chakrapani, S.; Roux, B.; Perozo, E. Structural basis for the coupling between activation and inactivation gates in $\mathrm{K}^{+}$channels. Nature 2010, 466, 272-275. [CrossRef]

88. Gibor, G.; Yakubovich, D.; Rosenhouse-Dantsker, A.; Peretz, A.; Schottelndreier, H.; Seebohm, G.; Dascal, N.; Logothetis, D.E.; Paas, Y.; Attali, B. An inactivation gate in the selectivity filter of KCNQ1 potassium channels. Biophys. J. 2007, 93, 4159-4172. [CrossRef]

89. Xiong, W.; Li, R.A.; Tian, Y.; Tomaselli, G.F. Molecular motions of the outer ring of charge of the sodium channel: Do they couple to slow inactivation? J. Gen. Physiol. 2003, 122, 323-332. [CrossRef]

90. Abderemane-Ali, F.; Findeisen, F.; Rossen, N.D.; Minor, D.L., Jr. A Selectivity Filter Gate Controls Voltage-Gated Calcium Channel Calcium-Dependent Inactivation. Neuron 2019, 101, 1134-1149.e3. [CrossRef]

91. Twomey, E.C.; Yelshanskaya, M.V.; Grassucci, R.A.; Frank, J.; Sobolevsky, A.I. Channel opening and gating mechanism in AMPA-subtype glutamate receptors. Nature 2017, 549, 60-65. [CrossRef]

92. Ahern, C.A.; Payandeh, J.; Bosmans, F.; Chanda, B. The hitchhiker's guide to the voltage-gated sodium channel galaxy. J. Gen. Physiol. 2016, 147, 1-24. [CrossRef]

93. Koivisto, A.P.; Belvisi, M.G.; Gaudet, R.; Szallasi, A. Advances in TRP channel drug discovery: From target validation to clinical studies. Nat. Rev. Drug Discov. 2022, 21, 41-59. [CrossRef]

94. Korkosh, V.S.; Zaytseva, A.K.; Kostareva, A.A.; Zhorov, B.S. Intersegment Contacts of Potentially Damaging Variants of Cardiac Sodium Channel. Front. Pharmacol. 2021, 12, 3055. [CrossRef]

95. Korkosh, V.S.; Kiselev, A.M.; Mikhaylov, E.N.; Kostareva, A.A.; Zhorov, B.S. Atomic Mechanisms of Timothy Syndrome-Associated Mutations in Calcium Channel Cav1.2. Front. Physiol. 2019, 10, 335. [CrossRef]

96. Zaytseva, A.K.; Boitsov, A.S.; Kostareva, A.A.; Zhorov, B.S. Possible Interactions of Extracellular Loop IVP2-S6 with VoltageSensing Domain III in Cardiac Sodium Channel. Front. Pharmacol. 2021, 12, 742508. [CrossRef]

97. Ulmschneider, M.B.; Bagneris, C.; McCusker, E.C.; Decaen, P.G.; Delling, M.; Clapham, D.E.; Ulmschneider, J.P.; Wallace, B.A. Molecular dynamics of ion transport through the open conformation of a bacterial voltage-gated sodium channel. Proc. Natl. Acad. Sci. USA 2013, 110, 6364-6369. [CrossRef]

98. Berneche, S.; Roux, B. Molecular dynamics of the KcsA K ${ }^{+}$channel in a bilayer membrane. Biophys. J. 2000, 78, 2900-2917. [CrossRef]

99. Shrivastava, I.H.; Sansom, M.S. Simulations of ion permeation through a potassium channel: Molecular dynamics of KcsA in a phospholipid bilayer. Biophys. J. 2000, 78, 557-570. [CrossRef]

100. Jensen, M.O.; Jogini, V.; Borhani, D.W.; Leffler, A.E.; Dror, R.O.; Shaw, D.E. Mechanism of voltage gating in potassium channels. Science 2012, 336, 229-233. [CrossRef]

101. Noskov, S.Y.; Berneche, S.; Roux, B. Control of ion selectivity in potassium channels by electrostatic and dynamic properties of carbonyl ligands. Nature 2004, 431, 830-834. [CrossRef]

102. Allen, T.W.; Kuyucak, S.; Chung, S.H. Molecular dynamics study of the KcsA potassium channel. Biophys. J. 1999, 77, 2502-2516. [CrossRef]

103. Biggin, P.C.; Smith, G.R.; Shrivastava, I.; Choe, S.; Sansom, M.S. Potassium and sodium ions in a potassium channel studied by molecular dynamics simulations. Biochim. Biophys. Acta 2001, 1510, 1-9. [CrossRef]

104. Zhorov, B.S. Possible Mechanism of Ion Selectivity in Eukaryotic Voltage-Gated Sodium Channels. J. Phys. Chem. B 2021, 125, 2074-2088. [CrossRef]

105. Corry, B.; Thomas, M. Mechanism of ion permeation and selectivity in a voltage gated sodium channel. J. Am. Chem. Soc. 2012, 134, 1840-1846. [CrossRef]

106. Flood, E.; Boiteux, C.; Allen, T.W. Selective ion permeation involves complexation with carboxylates and lysine in a model human sodium channel. PLoS Comput. Biol. 2018, 14, e1006398. [CrossRef]

107. Chakrabarti, N.; Ing, C.; Payandeh, J.; Zheng, N.; Catterall, W.A.; Pomes, R. Catalysis of $\mathrm{Na}^{+}$permeation in the bacterial sodium channel Na(V)Ab. Proc. Natl. Acad. Sci. USA 2013, 110, 11331-11336. [CrossRef]

108. Gomez-Lagunas, F.; Armstrong, C.M. The relation between ion permeation and recovery from inactivation of ShakerB $\mathrm{K}^{+}$ channels. Biophys. J. 1994, 67, 1806-1815. [CrossRef]

109. Hoshi, T.; Zagotta, W.N.; Aldrich, R.W. Biophysical and molecular mechanisms of Shaker potassium channel inactivation. Science 1990, 250, 533-538. [CrossRef] 
110. Zagotta, W.N.; Hoshi, T.; Aldrich, R.W. Restoration of inactivation in mutants of Shaker potassium channels by a peptide derived from ShB. Science 1990, 250, 568-571. [CrossRef]

111. Tikhonov, D.B.; Zhorov, B.S. The pore domain in glutamate-gated ion channels: Structure, drug binding and similarity with potassium channels. Biochim. Biophys. Acta Biomembr. 2020, 1862, 183401. [CrossRef]

112. Stevens, M.; Peigneur, S.; Tytgat, J. Neurotoxins and their binding areas on voltage-gated sodium channels. Front. Pharmacol. 2011, 2, 71. [CrossRef]

113. Catterall, W.A.; Swanson, T.M. Structural Basis for Pharmacology of Voltage-Gated Sodium and Calcium Channels. Mol. Pharmacol. 2015, 88, 141-150. [CrossRef]

114. Catterall, W.A. Sodium channels, inherited epilepsy, and antiepileptic drugs. Annu. Rev. Pharmacol. Toxicol. 2014, 54, 317-338. [CrossRef]

115. Silver, K.S.; Du, Y.; Nomura, Y.; Oliveira, E.E.; Salgado, V.L.; Zhorov, B.S.; Dong, K. Voltage-Gated Sodium Channels as Insecticide Targets. Adv. Insect Phys. 2014, 46, 389-433. [CrossRef]

116. Korkosh, V.S.; Zhorov, B.S.; Tikhonov, D.B. Folding similarity of the outer pore region in prokaryotic and eukaryotic sodium channels revealed by docking of conotoxins GIIIA, PIIIA, and KIIIA in a NavAb-based model of Nav1.4. J. Gen. Physiol. 2014, 144, 231-244. [CrossRef]

117. Tomasic, T.; Hartzoulakis, B.; Zidar, N.; Chan, F.; Kirby, R.W.; Madge, D.J.; Peigneur, S.; Tytgat, J.; Kikelj, D. Ligand- and structure-based virtual screening for clathrodin-derived human voltage-gated sodium channel modulators. J. Chem. Inf. Model. 2013, 53, 3223-3232. [CrossRef]

118. Palestro, P.H.; Enrique, N.; Goicoechea, S.; Villalba, M.L.; Sabatier, L.L.; Martin, P.; Milesi, V.; Bruno Blanch, L.E.; Gavernet, L. Searching for New Leads to Treat Epilepsy: Target-Based Virtual Screening for the Discovery of Anticonvulsant Agents. J. Chem. Inf. Model. 2018, 58, 1331-1342. [CrossRef]

119. Tikhonov, D.B.; Bruhova, I.; Zhorov, B.S. Atomic determinants of state-dependent block of sodium channels by charged local anesthetics and benzocaine. FEBS Lett. 2006, 580, 6027-6032. [CrossRef]

120. Bruhova, I.; Tikhonov, D.B.; Zhorov, B.S. Access and binding of local anesthetics in the closed sodium channel. Mol. Pharmacol. 2008, 74, 1033-1045. [CrossRef] [PubMed]

121. Hille, B. Local anesthetics: Hydrophilic and hydrophobic pathways for the drug-receptor reaction. J. Gen. Physiol. 1977, 69, 497-515. [CrossRef] [PubMed]

122. Buyan, A.; Sun, D.; Corry, B. Protonation state of inhibitors determines interaction sites within voltage-gated sodium channels. Proc. Natl. Acad. Sci. USA 2018, 115, E3135-E3144. [CrossRef] [PubMed]

123. Martin, L.J.; Corry, B. Locating the route of entry and binding sites of benzocaine and phenytoin in a bacterial voltage gated sodium channel. PLoS Comput. Biol. 2014, 10, e1003688. [CrossRef] [PubMed]

124. Tikhonov, D.B.; Zhorov, B.S. Mechanism of sodium channel block by local anesthetics, antiarrhythmics, and anticonvulsants. J. Gen. Physiol. 2017, 149, 465-481. [CrossRef]

125. Nguyen, P.T.; DeMarco, K.R.; Vorobyov, I.; Clancy, C.E.; Yarov-Yarovoy, V. Structural basis for antiarrhythmic drug interactions with the human cardiac sodium channel. Proc. Natl. Acad. Sci. USA 2019, 116, 2945-2954. [CrossRef]

126. Fozzard, H.A.; Lipkind, G.M. The tetrodotoxin binding site is within the outer vestibule of the sodium channel. Mar. Drugs 2010, 8, 219-234. [CrossRef]

127. Lipkind, G.M.; Fozzard, H.A. A structural model of the tetrodotoxin and saxitoxin binding site of the $\mathrm{Na}^{+}$channel. Biophys. J. 1994, 66, 1-13. [CrossRef]

128. Xu, L.; Li, D.; Ding, J.; Pan, L.; Ding, X. Insight into tetrodotoxin blockade and resistance mechanisms of Nav 1.2 sodium channel by theoretical approaches. Chem. Biol. Drug Des. 2018, 92, 1445-1457. [CrossRef]

129. Chen, R.; Chung, S.H. Binding modes of $\mu$-conotoxin to the bacterial sodium channel (NaVAb). Biophys. J. 2012, 102, 483-488. [CrossRef]

130. Mahdavi, S.; Kuyucak, S. Molecular dynamics study of binding of micro-conotoxin GIIIA to the voltage-gated sodium channel $\mathrm{Na}(\mathrm{v})$ 1.4. PLoS ONE 2014, 9, e105300. [CrossRef]

131. Zamponi, G.W.; Striessnig, J.; Koschak, A.; Dolphin, A.C. The Physiology, Pathology, and Pharmacology of Voltage-Gated Calcium Channels and Their Future Therapeutic Potential. Pharmacol. Rev. 2015, 67, 821-870. [CrossRef]

132. Godfraind, T. Discovery and Development of Calcium Channel Blockers. Front. Pharmacol. 2017, 8, 286. [CrossRef]

133. Cheng, R.C.; Tikhonov, D.B.; Zhorov, B.S. Structural model for phenylalkylamine binding to L-type calcium channels. J. Biol. Chem. 2009, 284, 28332-28342. [CrossRef] [PubMed]

134. Tikhonov, D.B.; Zhorov, B.S. Structural Model for Dihydropyridine Binding to L-type Calcium Channels. J. Biol. Chem. 2009, 284, 19006-19017. [CrossRef]

135. Tikhonov, D.B.; Zhorov, B.S. Molecular modeling of benzothiazepine binding in the L-type calcium channel. J. Biol. Chem. 2008, 283, 17594-17604. [CrossRef] [PubMed]

136. Li, W.; Shi, G. How CaV1.2-bound verapamil blocks Ca ${ }^{2+}$ influx into cardiomyocyte: Atomic level views. Pharmacol. Res. 2019, 139, 153-157. [CrossRef] [PubMed]

137. Tikhonov, D.B.; Bruhova, I.; Garden, D.P.; Zhorov, B.S. State-dependent inter-repeat contacts of exceptionally conserved asparagines in the inner helices of sodium and calcium channels. Pflug. Arch. 2015, 467, 253-266. [CrossRef] 
138. Du, Y.Z.; Tikhonov, D.B.; Nomura, Y.; Dong, K.; Zhorov, B.S. Mutational analysis of state-dependent contacts in the pore module of eukaryotic sodium channels. Arch. Biochem. Biophys. 2018, 652, 59-70. [CrossRef]

139. Tikhonov, D.B.; Zhorov, B.S. Conservation and variability of the pore-lining helices in P-loop channels. Channels 2017, 11, 660-672. [CrossRef]

140. Yelshanskaya, M.V.; Nadezhdin, K.D.; Kurnikova, M.G.; Sobolevsky, A.I. Structure and function of the calcium-selective TRP channel TRPV6. J. Physiol. 2021, 599, 2673-2697. [CrossRef]

141. Zubcevic, L.; Lee, S.Y. The role of pi-helices in TRP channel gating. Curr. Opin. Struct. Biol. 2019, 58, 314-323. [CrossRef]

142. Kokubun, S.; Prod'hom, B.; Becker, C.; Porzig, H.; Reuter, H. Studies on Ca channels in intact cardiac cells: Voltage-dependent effects and cooperative interactions of dihydropyridine enantiomers. Mol. Pharmacol. 1986, 30, 571-584.

143. Correa, A.M.; Bezanilla, F.; Latorre, R. Gating kinetics of batrachotoxin-modified $\mathrm{Na}^{+}$channels in the squid giant axon. Voltage and temperature effects. Biophys. J. 1992, 61, 1332-1352. [CrossRef]

144. Quandt, F.N.; Narahashi, T. Modification of single $\mathrm{Na}^{+}$channels by batrachotoxin. Proc. Natl. Acad. Sci. USA 1982, 79, $6732-6736$. [CrossRef]

145. Garber, S.S.; Miller, C. Single $\mathrm{Na}^{+}$channels activated by veratridine and batrachotoxin. J. Gen. Physiol. 1987, 89, 459-480. [CrossRef]

146. Tunyasuvunakool, K.; Adler, J.; Wu, Z.; Green, T.; Zielinski, M.; Zidek, A.; Bridgland, A.; Cowie, A.; Meyer, C.; Laydon, A.; et al. Highly accurate protein structure prediction for the human proteome. Nature 2021, 596, 590-596. [CrossRef]

147. Baek, M.; DiMaio, F.; Anishchenko, I.; Dauparas, J.; Ovchinnikov, S.; Lee, G.R.; Wang, J.; Cong, Q.; Kinch, L.N.; Schaeffer, R.D.; et al. Accurate prediction of protein structures and interactions using a three-track neural network. Science 2021, 373, 871-876. [CrossRef]

148. Buel, G.R.; Walters, K.J. Can AlphaFold2 predict the impact of missense mutations on structure? Nat. Struct. Mol. Biol. 2022, 29, 1-2. [CrossRef]

149. Meadows, L.S.; Isom, L.L. Sodium channels as macromolecular complexes: Implications for inherited arrhythmia syndromes. Cardiovasc. Res. 2005, 67, 448-458. [CrossRef]

150. Haworth, A.S.; Brackenbury, W.J. Emerging roles for multifunctional ion channel auxiliary subunits in cancer. Cell Calcium $\mathbf{2 0 1 9}$ 80, 125-140. [CrossRef]

151. Dolphin, A.C. Voltage-gated calcium channels and their auxiliary subunits: Physiology and pathophysiology and pharmacology. J. Physiol. 2016, 594, 5369-5390. [CrossRef]

152. Gonzalez-Perez, V.; Lingle, C.J. Regulation of BK Channels by Beta and Gamma Subunits. Annu. Rev. Physiol. 2019, 81, 113-137. [CrossRef]

153. Zhorov, B.S.; Du, Y.; Song, W.; Luo, N.; Gordon, D.; Gurevitz, M.; Dong, K. Mapping the interaction surface of scorpion beta-toxins with an insect sodium channel. Biochem. J. 2021, 478, 2843-2869. [CrossRef] 